\title{
Habitat selection of a parasitoid mediated by volatiles informing on host and intraguild predator densities
}

\author{
Belén Cotes ${ }^{1} \cdot$ Linda-Marie Rännbäck $^{1} \cdot$ Maria Björkman $^{2} \cdot$ Hans Ragnar Norli $^{2}$. \\ Nicolai V. Meyling ${ }^{3} \cdot$ Birgitta Rämert $^{1} \cdot$ Peter Anderson $^{4}$
}

Received: 14 January 2015 / Accepted: 16 April 2015 / Published online: 7 May 2015

(C) The Author(s) 2015. This article is published with open access at Springerlink.com

\begin{abstract}
To locate and evaluate host patches before oviposition, parasitoids of herbivorous insects utilize plant volatiles and host-derived cues, but also evaluate predatorderived infochemicals to reduce predation risks. When foraging in host habitats infested with entomopathogenic fungi that can infect both a parasitoid and its host, parasitoids may reduce the risk of intraguild predation (IGP) by avoiding such patches. In this study, we examined whether the presence of the entomopathogenic fungi Metarhizium brunneum and Beauveria bassiana in soil habitats of a root herbivore, Delia radicum, affects the behavior of Trybliographa rapae, a parasitoid of $D$. radicum. Olfactometer bioassays revealed that $T$. rapae avoided fungal infested host habitats and that this was dependent on fungal species and density. In particular, the parasitoid avoided habitats
\end{abstract}

Communicated by Maria J. Pozo.

Electronic supplementary material The online version of this article (doi:10.1007/s00442-015-3326-2) contains supplementary material, which is available to authorized users.

Belén Cotes

Belen.cotes@slu.se

1 Department of Plant Protection Biology, Integrated Plant Protection, Swedish University of Agricultural Sciences, P.O. Box 102, Växtskyddsvägen 3, Alnarp SE-230 53, Sweden

2 Plant Health and Plant Protection Division, Norwegian Institute for Agricultural and Environmental Research (Bioforsk), Høgskoleveien 7, Ås 1430, Norway

3 Department of Plant and Environmental Sciences, University of Copenhagen, Thorvaldsensvej 40, Frederiksberg C 1871, Denmark

4 Department of Plant Protection Biology, Chemical Ecology, Swedish University of Agricultural Sciences, P.O. Box 102, Växtskyddsvägen 3, Alnarp SE-230 53, Sweden with high densities of the more virulent fungus, M. brunneum. In addition, host density was found to be important for the attraction of T. rapae. Volatiles collected from host habitats revealed different compound profiles depending on fungal presence and density, which could explain the behavior of $T$. rapae. We conclude that $T$. rapae females may use volatile compounds to locate high densities of prey, but also compounds related to fungal presence to reduce the risk of IGP towards themselves and their offspring.

Keywords Delia radicum - Entomopathogenic fungus . Foraging decision · Trade-off · Trybliographa rapae

\section{Introduction}

When two species which share a common prey resource interact with each other in terms of competition and predation per se this is known as intraguild predation (IGP) (Polis et al. 1989). The interaction can be either unidirectional or bidirectional and, in the latter case, the roles as IG predator and IG prey can shift. An example of a unidirectional IGP system is when a pathogen can infect both a herbivore and its parasitoid (Rosenheim et al. 1995; Straub et al. 2008).

Natural enemies of herbivores can utilize volatile secondary metabolites emitted by plants attacked by herbivores, i.e., herbivore-induced plant volatiles (HIPVs), when searching for prey (Dicke and Baldwin 2010). HIPVs and other host-derived cues have been shown to guide female parasitoids to locate and evaluate host patches before oviposition (Vet and Dicke 1992; Wäschke et al. 2013). It is also well established that host density plays a role when parasitoid females select suitable habitats for oviposition 
(Waage 1983; Connor and Cargain 1994; Hemachandra et al. 2007; Girling et al. 2011). The mechanism involved may be an increase in emitted HIPVs with higher host densities (Girling et al. 2011). However, parasitoid host foraging assumes trade-offs between oviposition sites (i.e., expected fitness gain) and the risk of predation (Weisser et al. 1994; Roitberg et al. 2010). In addition to host-derived cues, parasitoids may also respond to predator-derived infochemicals and avoid predation risks by diverting foraging to "safer" habitats (Raymond et al. 2000; Dicke and Grostal 2001; Nakashima et al. 2004; Meisner et al. 2011). Thus, by exhibiting variable anti-predator behavior, parasitoids can partly or fully avoid the potential disruptive impact of IGP (Snyder and Ives 2008).

Generalist entomopathogenic fungi can act as IG predators, as they infect insects at different trophic levels (Brooks 1993; Roy and Pell 2000; Furlong and Pell 2005). Entomopathogenic fungi are also known to affect the behavior of insects in different ways (Roy et al. 2006; Baverstock et al. 2010) and predators have been found to perceive and avoid fungal infested habitats, as well as infected prey and conspecifics (Meyling and Pell 2006; Ormond et al. 2011). Parasitoids may also be able to evaluate the IGP risk when foraging in fungal infested host habitats. Upon encountering fungal infected hosts in choice situations, both avoidance (Brobyn et al. 1988; Fransen and van Lenteren 1993) as well as a lack of avoidance (Lord 2001; Baverstock et al. 2005) have been demonstrated for different parasitoid species. During growth, fungi produce volatile metabolites (Crespo et al. 2008), which can be used as infochemicals by insects to avoid unsuitable habitats (Mburu et al. 2013). Furthermore, it is increasingly recognized that the host-habitat-selection behavior of natural enemies of herbivores can also be influenced by rhizosphere-inhabiting microbes via induced changes in the composition of released HIPVs (Guerrieri et al. 2004; Schausberger et al. 2012; Battaglia et al. 2013; Pineda et al. 2013; Soler et al. 2013).

In a host-pathogen-parasitoid system we studied the habitat choice of the wasp Trybliographa rapae Westwood (Hymenoptera: Figitidae), an oligophagous, solitary parasitoid of the below-ground larval stages of several Delia spp. (Diptera: Anthomyiidae) (Wishart and Monteith 1954). We used larvae of the cabbage root fly, Delia radicum L., a herbivore of roots of cruciferous plants in temperate climates (Finch 1989), feeding on white cabbage plants, as a model host habitat. Host habitat location by T. rapae females is mainly guided by olfactory cues with HIPVs released by the plant in combination with host-related cues (e.g., saliva, feces) informing the parasitoid of host presence (Brown and Anderson 1999; Neveu et al. 2002; Nilsson et al. 2012) and host density (Hemachandra et al. 2007). As pathogens, we used the generalist entomopathogenic fungi Metarhizium brunneum Petch and Beauveria bassiana (Balsamo) Vuillemin (Ascomycota: Hypocreales), which are ubiquitous in the soil environment (Meyling and Eilenberg 2007) and occur naturally at higher densities in plant rhizospheres than in the surrounding soil (Bruck 2010). These fungi can infect both $D$. radicum and T. rapae (Rännbäck et al. 2015), but it is currently unknown to what extent fungal presence in the host habitat influences foraging decisions by T. rapae females to reduce the risk of unidirectional IGP.

In the present study we addressed the following questions:

1. Do T. rapae females avoid D. radicum host habitats infested by different species of generalist entomopathogenic fungi?

2. If avoidance is observed, is this dependent on the densities of host larvae and fungus?

3. Is parasitoid selection in this system linked to changes in the composition of volatile emissions from the host habitats?

\section{Materials and methods}

\section{Insect and plant material}

The $D$. radicum culture used in the study originated from a laboratory culture at Warwick Crop Centre, Wellesbourne, UK, and was reared following the method described by Finch and Coaker (1969). The T. rapae culture originated from a laboratory culture from the University of Rennes, France, and the rearing method was modified from Neveu et al. (1996). In both insect cultures, we introduced fieldcollected insects from Sweden every year. The adult wasps and flies were kept in separate cabinets at $19^{\circ} \mathrm{C}$ and a constant photoperiod of 16-h light:8-h dark (L:D). We provided the adult parasitoids with sucrose water and honey droplets and the adult flies with a mixture of honey, dried yeast, and milk powder, replacing the food twice a week. Female parasitoids used in the experiments were separated according to age (day of emergence), provided sucrose water and kept in rearing cabinets with males for mating.

We sowed seeds of white cabbage (Brassica oleracea var. capitata f. alba cv. Castello) in potting soil (Hasselfors Garden Special; Hasselfors Garden, Sweden) mixed with a slow-release fertilizer (Osmocote Pro 3-4 months, nitrogen-phosphorous-potassium 17-11-10 plus micronutrients; Weibulls Horto, Sweden) in 1.5- and 3.0-L plastic pots. The pots were then kept in a greenhouse chamber $\left[22 \pm 1{ }^{\circ} \mathrm{C}, 75 \%\right.$ relative humidity (RH), 16L:8D photoperiod] and the plants were used in experiments when they had eight and ten true leaves, corresponding to an age of 2 months. 


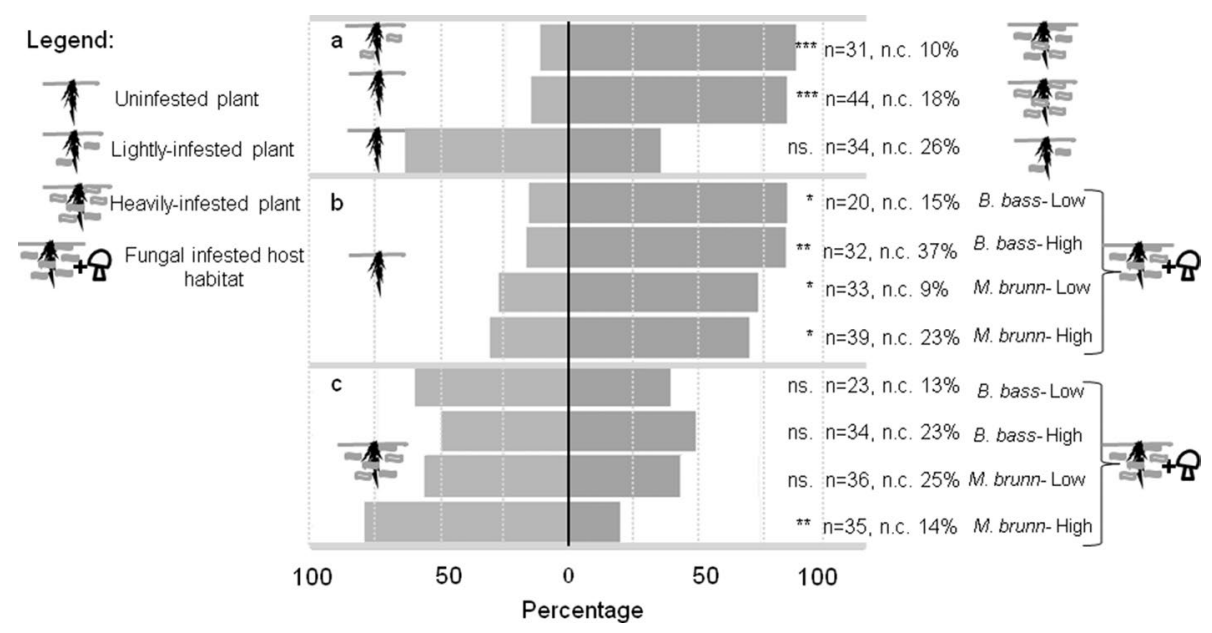

Fig. 1 Behavioral response of Trybliographa rapae females offered a different infestation densities of Delia radicum larvae, $\mathbf{b}$ uninfested plants (UP) and heavily-infested plants (HIP) with low or high density of Beauveria bassiana or Metarhizium brunneum, and c HIP and HIP with low or high density of B. bassiana or M. brunneum.

Plant infestation involved placing 15 or $30 \mathrm{D}$. radicum eggs around the stem base of the plant, and covering them with damp soil, 9 days prior to the fungal inoculation. Since the egg hatching rate was not $100 \%$ we established two levels of infestation: lightly infested plants (LIP; from one to ten larvae) or heavily infested plants (HIP; 15-30 larvae). Plants with 11-14 larvae were excluded from the analysis and uninfested plants (UP) used as controls. At the end of each experiment, we recorded the number of larvae on each plant.

\section{Fungal inoculation of plants}

Two fungal isolates of $M$. brunneum (isolate KVL 04-57) and $B$. bassiana (isolate KVL 03-90), stored at $-80{ }^{\circ} \mathrm{C}$ at the University of Copenhagen, Department of Plant and Environmental Sciences, were used in this study. For the preparation of cultures and conidial suspensions, we followed the methods described by Rännbäck et al. (2015). Concentrations of undiluted stock suspensions were established by serial dilutions and enumerations in a hemocytometer $\left(0.0625 \mathrm{~mm}^{2}\right.$, depth $0.200 \mathrm{~mm}$; FuchsRosenthal, VWR, Sweden). To determine conidia viability, we assessed the proportion of germinated conidia under $400 \times$ magnification after 24 h growth on Sabouraud dextrose agar. Germination was always more than $95 \%$. All stock suspensions were used on the day after preparation, at which time the fungal suspensions for the experiments were prepared.

We performed fungal inoculation of the plants $48 \mathrm{~h}$ prior to the experiments by manually pouring the fungal suspension around the stem base, in close proximity to
Test on two-tailed binomial distribution, ${ }^{*} P<0.05$, $* * P<0.01$, $* * * P<0.001, n s$ not significant, $n$ numbers of female responders, n.c. percentages of female responders not making a choice (excluded from the statistical analysis)

the actively feeding $D$. radicum larvae. The HIP in 3.0or 1.5 -L pots received 40 or $20 \mathrm{~mL}$ of fungal suspension, respectively. To flush the conidia further down the root system, 20 or $10 \mathrm{~mL}$, respectively, of tap water was added around the stem base immediately after the application of the fungal suspension. The same volumes of tap water were used for UP pots. We established two different densities of B. bassiana (B. bassiana-High, $1 \times 10^{9}$ conidia $\mathrm{mL}^{-1} ; B$. bassiana-Low, $1 \times 10^{8}$ conidia $\mathrm{mL}^{-1}$ ) and $M$. brunneum (M. brunneum-High, $1 \times 10^{8}$ conidia $\mathrm{mL}^{-1} ;$ M. brunneum-Low, $5 \times 10^{7}$ conidia $\mathrm{mL}^{-1}$ ). These densities were based on a pilot study, in which the low densities resulted in $<20 \%$ larval mortality and the high densities resulted in $>50 \%$ larval mortality in D. radicum, as reported previously by Chandler and Davidson (2005) for Metarhizium anisopliae. As a lower inoculum level was required for $M$. brunneum compared with B. bassiana to achieve this effect, we assumed that the different fungal densities resulted in comparable infection levels of the two fungal species in $D$. radicum larvae.

\section{Olfactometer bioassay}

We evaluated the responses of host-naïve $T$. rapae females to host density and fungal infested habitats in an olfactometer set-up as described by Jönsson et al. (2005). For host density responses, we tested three treatment combinations: UP vs. LIP, UP vs. HIP, and LIP vs. HIP (Fig. 1a). For responses to fungal infested habitats, the tested combinations were UP vs. HIP with $B$. bassiana-High, $B$. bassianaLow, M. brunneum-High and M. brunneum-Low (Fig. 1b) and HIP vs. the same four fungal treatments (Fig. 1c). 
All experiments were conducted in a climate chamber at $24{ }^{\circ} \mathrm{C}, 60 \% \mathrm{RH}$ and a light intensity of 1300 lux (measured at the Y-tube intersection), between 8 a.m. and 5 p.m. The air introduced into the set-up was pumped through a bottle $(250 \mathrm{~mL})$ with activated charcoal to eliminate surrounding odors, and then through a bottle with water to provide humidity (7-Ah, 12-V Micropump; KNF Neuberger, Germany). We adjusted the air flow rate to each arm of the olfactometer to $0.8 \pm 0.2 \mathrm{~L} \mathrm{~min}^{-1}$ by a flow meter and then allowed the parasitoids to acclimatize to room conditions for at least $30 \mathrm{~min}$ before being tested in the olfactometer. A piece of black plastic tube $(2 \mathrm{~cm})$ covered the arm $1 \mathrm{~cm}$ from the junction of the Y-shaped glass tube [arm length $220 \mathrm{~mm}, 15 \mathrm{~mm}$ inner diameter (ID)], in order to calm the insect before it chose a direction. We tested parasitoids individually and recorded a choice when they moved $5 \mathrm{~cm}$ into one of the arms and stayed there for no less than $30 \mathrm{~s}$. Parasitoids which did not make a choice within $5 \mathrm{~min}$ were discarded and excluded from the statistical analysis. After tests on five individuals we shifted the position of the stimuli in the olfactometer, in order to eliminate potential positional bias. Moreover, after ten individuals had been tested we renewed the Y-tube stimuli source. Thus, each replication, which consisted of ten females, lasted $<2 \mathrm{~h}$. After use, all corks, Teflon tubes and Y-tubes were rinsed with ethanol $(70 \%)$ and sterile water and the Y-tube was heated in an oven at $400{ }^{\circ} \mathrm{C}$ for $8 \mathrm{~h}$ between experimental days. We prepared the odor stimuli by carefully removing the plants (including soil) from the pot and placing them in a polyacetate cooking bag $(35 \times 43 \mathrm{~cm})$ (Toppits; Melitta), attached tightly with tape to the olfactometer. Plants grown in different-sized pots $(1.5$ and $3 \mathrm{~L})$ were tested in order to check whether stress in root system growth could influence parasitoid choice.

\section{Volatile collection}

To collect volatiles, we carefully removed an entire plant (including soil) from the pot, placed it in the same type of cooking bag used in the olfactometer experiments and sealed it to the Teflon tubes (ThermoFisher Scientific, Santa Clara, CA) of the collection set-up with tape. Volatile collection involved the following seven treatments (only 3-L pots): empty bags, soil, UP, HIP, and HIP with B. bassiana-High, M. brunneum-High, and M. brunneum-Low, with five replicate plants for HIP and eight for the other treatments.

The volatile collection system comprised a tandem Teflon filter holder, so that the sampled odors passed through each adsorbent filter in sequence. Each Teflon tube was $25 \mathrm{~mm}$ long with $3 \mathrm{~mm}$ ID and the front filter contained $15 \mathrm{~mm}$ of Tenax GR (80-60 mesh) (Scantec; Lab AB, Partille, Sweden) and the second filter $15 \mathrm{~mm}$ Porapak-Q
(80-100 mesh) (Scantec; Lab AB). Each filter tube was held by two stoppers of Teflon tube ( $2 \mathrm{~mm}$ ID) and glass wool. We inserted the filters into the polyacetate bags, placed a charcoal filter in the opposite corner to purify incoming air and drew out volatiles using a reversed aquarium Rena 300 pump (Mars Fishcare, Chalfont, PA) at a flow rate through the filters of $100 \mathrm{~mL}$ air $\mathrm{min}^{-1}$ before starting the experiment, a value measured and adjusted by a flow meter. Volatile collection continued for $4 \mathrm{~h}$, under the same climate chamber conditions as in the olfactometer experiments.

For analysis, we eluted each pair of adsorbent filters in individual GC vials with $500 \mu \mathrm{L}$ hexane (purity $\geq 99 \%$; Fluka, Buchs, Switzerland). Each GC vial received $2 \mu \mathrm{L}$ of an internal standard mixture containing $250 \mathrm{ng} \mu \mathrm{L}^{-1}$ heptyl acetate and $250 \mathrm{ng} \mu \mathrm{L}^{-1}$ undecyl acetate and then all vials were capped and stored at $-20{ }^{\circ} \mathrm{C}$ until analysis. Before gas chromatography-mass spectrometry (GC-MS) analysis, the samples were concentrated with a stream of nitrogen to approximately $30-40 \mu \mathrm{L}$.

\section{Chemical analysis and compound identification}

The system used for sample analysis comprised an Agilent 6890 N GC connected to an Agilent 5973 MS and a deconvolution reporting system (version A.02.00; Agilent Technologies) for compound identification. The method for compound analysis and identification is identical to that described in detail by Thöming et al. (2014). The identity of six selected compounds [dimethyl disulfide, $\beta$-myrcene, $o$-xylene, 3-hexanol, (Z)-3-hexen-1-yl-acetate and 1,3-ditert-butylbenzene] was verified by comparing mass spectra, Kovats index, and retention time with those obtained for synthetic standards (Sigma-Aldrich, Zwijndrecht, the Netherlands) on the same column. Relative amounts of identified compounds were calculated by dividing the peak area (using the area from the total ion chromatogram) by the area of the internal standard heptyl acetate. Only compounds found exclusively in treatments with $D$. radicum-infested plants were analyzed further, i.e., we excluded all compounds found in control samples (empty bags and soil) and UP.

\section{Statistical analysis}

Data analysis began with data exploration, following the protocol described by Zuur et al. (2010) and using R software ( $R$ Core Team 2012). The first analysis examined the female responses of every single treatment combination (Fig. 1) to determine whether there was a preference for one of the two odor sources. A two-tailed binomial test was applied with the null hypothesis that the distribution of the parasitoids over the two arms of the olfactometer was 50:50. 
Table 1 Covariates included in the Bernoulli generalized linear model for Trybliographa rapae behavioral choices

\begin{tabular}{|c|c|c|c|}
\hline \multirow{2}{*}{$\begin{array}{l}\text { Response variable } \\
\begin{array}{l}\text { Difference in larval } \\
\text { density }\end{array}\end{array}$} & \multicolumn{2}{|c|}{$\begin{array}{l}\text { Abbreviation in predictorType of response variable } \\
\text { function }\end{array}$} & \multirow{2}{*}{$\begin{array}{l}\text { Description } \\
\text { Number of Delia radicum larvae in heavily infested plants (HIP) } \\
\text { with fungus minus number of larvae in non-infested plants } \\
\text { [uninfested plants (UP) or HIP] }\end{array}$} \\
\hline & Larvae $\operatorname{dif}_{i}$ & Continuous & \\
\hline Searching time & Search time $_{i}$ & Continuous & Time (min) spent in one arm after crossing the border of $5 \mathrm{~cm}$ \\
\hline Fungal treatments & Fungal treatment $_{i}$ & Factor (four levels) & $\begin{array}{l}\text { Beauveria bassiana-High, } \text { B. bassiana-Low, } \\
\text { Metarhizium brunneum-High, M. brunneum-Low }\end{array}$ \\
\hline Arm orientation & Arm orientation $_{i}$ & Factor (two levels) & Right and left (arm orientation) \\
\hline Pot size & 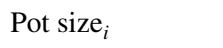 & Factor (two levels) & 1.5- and 3.0-L pots \\
\hline
\end{tabular}

The second analysis focused on factors that might have affected the response of parasitoids among all the different treatment combinations which included fungi (Fig. 1b, c). We modeled the probability of choosing a fungal infested host habitat $\left(\pi_{i}\right)$ as a function of several covariates by fitting a Bernoulli generalized linear model (GLM) with logistic link function $\log (\pi i)=\eta_{i}$. We assume that the $N_{i}$ female parasitoids are independent, because each parasitoid in group $i$ has the probability $\pi_{i}$. The covariates used in the predictor function $\left(\eta_{i}\right)$ were two continuous variables (searching time and difference in larval density) and three factors (fungal treatment, arm orientation and pot size) as main terms (Table 1). Thus, the predictor function was written as follows:

$$
\begin{aligned}
\eta_{i}=\text { Intercept }+ \text { Search Time }_{i}+\text { Larvae Dif }_{i} \\
\quad+\text { Fungal Treatment }_{i}+\text { Arm Orientation }_{i}+\text { Potsize }_{i}
\end{aligned}
$$

and this model was built in a Bayesian framework using JAGS software (Plummer 2008), and implemented in the R2jags package ( $\mathrm{Su}$ and Yajima 2012). A Bayesian approach has been suggested to describe how parasitoids forage in a patchy environment (Pierre and Green 2008).

We ran our model with 50,000 iterations and three chains in the Markov chain Monte Carlo (MCMC) process and dropped the first 40,000 iterations as the burn-in period. A thinning rate of ten based on initial checks for convergence, resulted in 3000 observations for calculating the posterior distributions. The forward selection procedure was used as model selection and the deviance information criterion (DIC) (Spiegelhalter et al. 2002) was used to choose the model best explaining the factors influencing habitat choice by $T$. rapae females. Models with smaller DICs are preferable. Finally, we estimated the posterior parameter distributions and observed covariate data to predict the mean response for the fitted model and obtain $99 \%$ confidence intervals for the fit.

Finally, we applied a principal component analysis (PCA) ordination technique to the relative amounts of identified compounds to graphically represent multivariate patterns in volatile emissions for plant treatments with or without fungi for different fungal species and fungal densities. Before the analysis was carried out, we normalized the variables and removed highly correlated compounds using variance inflation factors to assess which compounds were collinear (Montgomery and Peck 1992).

The PCA was based on the Euclidian distance between observations (scaling two) and the results displayed graphically in a PCA distance biplot using the vegan package (Oksanen et al. 2013). To assess differences in the volatiles between treatments, we used permutational multivariate ANOVA (PerMANOVA), which employs a permutation procedure to assess significance and thus does not rely on the assumption of multivariate normality (Anderson 2001).

We tested the effect of the treatments using ANOVA on peak areas for each compound using the Kruskal-Wallis non-parametric test and Bonferroni-corrected Mann-Whitney $U$-tests for post hoc comparisons.

\section{Results}

\section{Choice of host habitat}

The responses of $T$. rapae to volatiles emitted from $D$. radicum-infested habitats depended on the host densities of the plant pairs. Females were significantly attracted to volatiles from HIP compared with UP and LIP. However, the responses of the parasitoids were not significantly different when they were offered UP and LIP (Fig. 1a). When T. rapae encountered UP versus HIP with any fungus, females significantly preferred the plants with host odors, despite the presence of the fungus. This response was independent of fungal species or fungal density (Fig. 1b). On choosing between HIP and HIP with fungus added, females showed a significant avoidance response only for $M$. brunneum-High. Neither $B$. bassiana at any density nor $M$. brunneum-Low affected the choice by $T$. rapae between HIP and HIP with fungus (Fig. 1c). 
Table 2 Results of the Bernoulli generalized linear model for avoidance $(0)$ or attraction $(1)$ of $T$. rapae parasitoids towards highly $D$. radicuminfested plants with fungus, for a model including difference in larval density, fungal species and fungal densities

\begin{tabular}{lclllll}
\hline & Estimate & SE & $95 \%$ CI & $99 \%$ CI & $99.5 \%$ CI & Significance \\
\hline Intercept & 1.08 & 0.33 & $0.451,1.733$ & $0.317,1.906$ & $0.228,2.030$ & $* * *$ \\
Difference in larval density & 0.99 & 0.17 & $0.666,1.316$ & $0.607,1.373$ & $0.572,1.400$ & $* * *$ \\
M. brunneum-High & -1.24 & 0.44 & $-2.118,-0.379$ & $-2.283,-0.175$ & $-2.385,-0.063$ & $* *$ \\
M. brunneum-Low & -0.93 & 0.45 & $-1.803,-0.069$ & $-1.990,0.091$ & $-2.146,0.253$ & $*$ \\
B. bassiana-Low & -0.20 & 0.45 & $-1.129,0.664$ & $-1.294,0.846$ & $-1.404,0.963$ & NS \\
\hline
\end{tabular}

M. brunneum-High Plants inoculated with $1 \times 10^{8}$, M. brunneum-Low plants inoculated with $5 \times 10^{7}$ conidia of M. brunneum $\mathrm{mL}^{-1}$, Beauveria bassiana-Low plants inoculated with $1 \times 10^{8}$ conidia of $B$. bassiana $\mathrm{mL}^{-1}, C I$ confidence interval

NS $95 \%$ CI spans zero, * $95 \%$ CI does not span zero, ** $99 \%$ CI does not span zero, *** $99.5 \%$ CI does not span zero

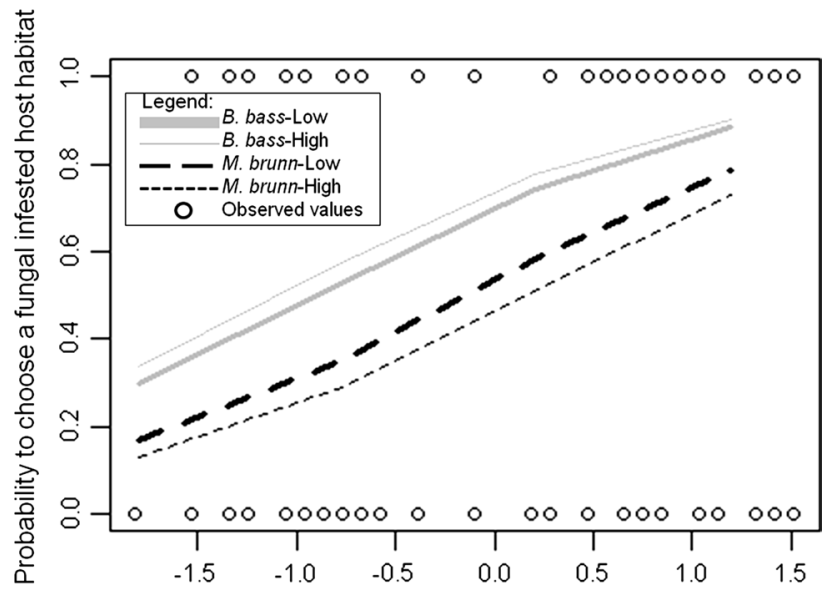

Standardized difference in larval density between pairs of plants (D. radicum infested plant $-D$. radicum infested plant with fungus)

Fig. 2 Parasitoid choice of host habitat in relation to host density and fungal species and density. The $x$-axis shows the standardized difference in larval density between pairs of plants (negative values signify higher larval densities in $D$. radicum-infested plants without fungi and positive values indicate higher densities in D. radicum-infested plants with fungi). The $y$-axis shows the probability of choosing a fungal infested host habitat (proportions of 0.5 equal attractions). The lines represent the fitted values for the four fungal treatments obtained by a Bernoulli generalized linear model applied to model 7 and open circles are observed values. Parasitoids are attracted to fungal infested host habitats with high densities of $D$. radicum larvae, but the attraction is higher in the presence of $B$. bassiana-Low and B. bassiana-High (grey lines) compared with M. brunneum-Low and M. brunneum-High (black lines)

\section{Factors affecting choice of host habitat with fungal presence}

In the Bernoulli GLM we evaluated whether the distribution of choices of $T$. rapae among all the different treatment combinations was significantly affected by the factors listed in Table 1, i.e., which covariates influenced parasitoid responses. The response values were: avoidance (values 0) or attraction (values 1) towards a HIP with fungus.
We compared the first ten potential models including only main terms based on their DIC values (Online Resource 1). Model 9 had the smallest DIC, but model 7 had only a slightly higher DIC. Due to the small difference between these two values of DIC, we selected the simpler model 7 for model interpretation. Furthermore, model 7 , including differences in larval density and fungal treatment, showed a good mixing of chains with the three lines of chains hovering around equilibrium (Online Resource 2).

In addition to findings in Fig. $1 \mathrm{~b}$ and $\mathrm{c}$, where the treatment combinations are analyzed individually, the results of the Bernoulli GLM (model 7) showed that differences in density of $D$. radicum larvae between plant pairs significantly influenced parasitoid choice, independently of fungal species or fungal density (Table 2). The model results also showed that, despite the larval density effect, the attraction to HIP with fungus was significantly different depending on fungal species and fungal density. Table 2 compares the three fungal treatments: $M$. brunneum-High, $M$. brunneum-Low and $B$. bassiana-Low with reference to $B$. bassiana-High, which is absent since it is the baseline factor. In the presence of M. brunneum-High, the probability of attraction towards a fungal infested habitat was significantly lower than when $T$. rapae encountered $B$. bassiana-High. This effect was less significant in the presence of $M$. brunneum-Low and non-significant in the presence of $B$. bassiana-Low. Figure 2 provides a visual interpretation of model 7 . The results of the models $2-5$ with all the individual factors (Online Resource 3) showed that, in addition to difference in larval density, fungal species or fungal density, only arm orientation had a significant effect on determining the parasitoid's choice, when it was analyzed individually.

\section{Plant volatiles}

Analysis of the volatiles trapped from HIP and HIP with fungus using multivariate statistics (after removing compounds found in UP, empty bags and soil) revealed 14 


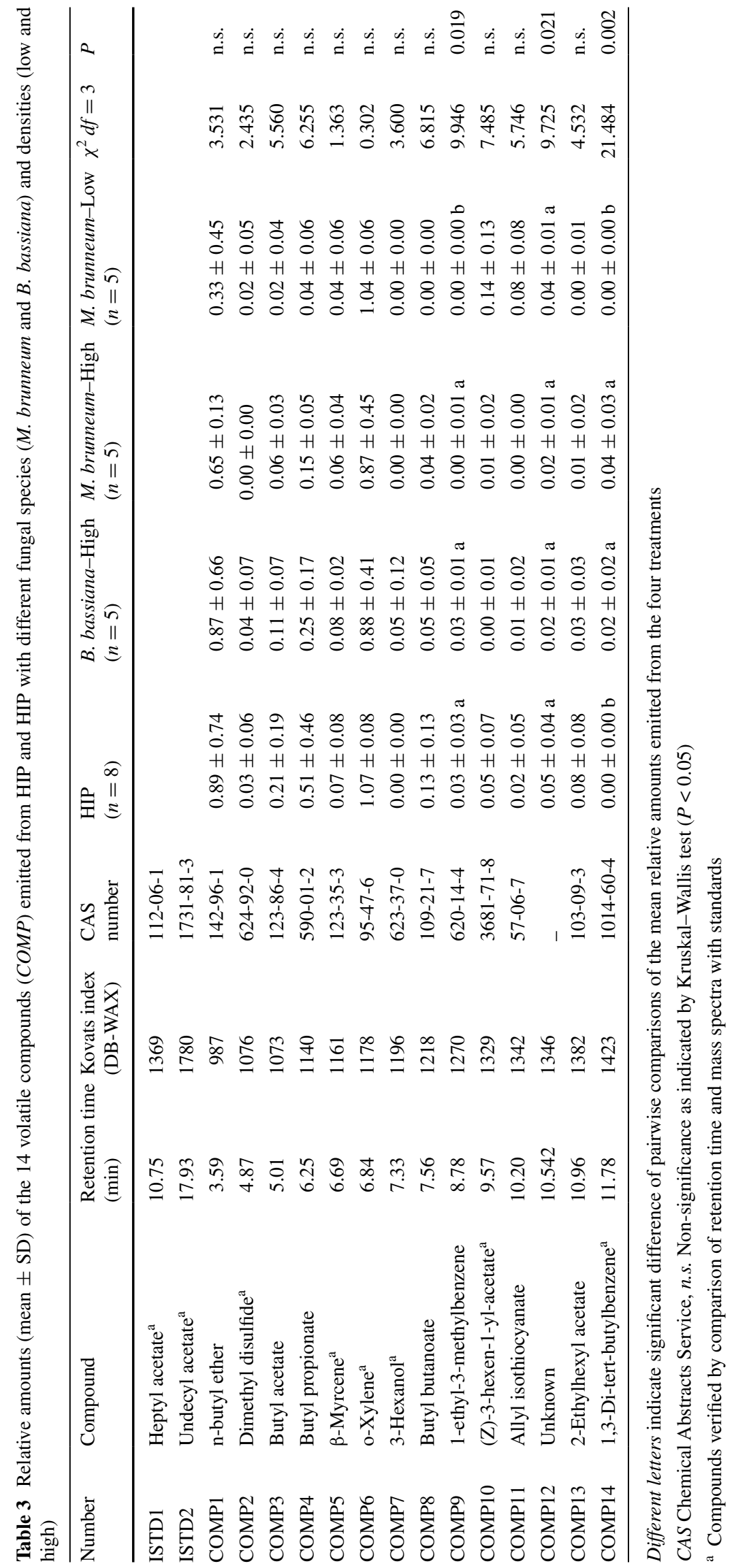


Fig. 3 Principal component (PC) analysis biplot for nine volatile compounds identified by gas chromatography-mass spectrometry (lines) and the 23 plant samples. Represented treatments are: HIP (circles); plants heavily infested with a high density of $B$. bassiana (B. bassiana-High; triangles); plants heavily infested with a low density of $M$. brunneum (M. brunneum-Low; grey stars); and plants heavily infested with a high density of $M$. brunneum (M. brunneum-High; black stars). The PCs are PC1 and PC2

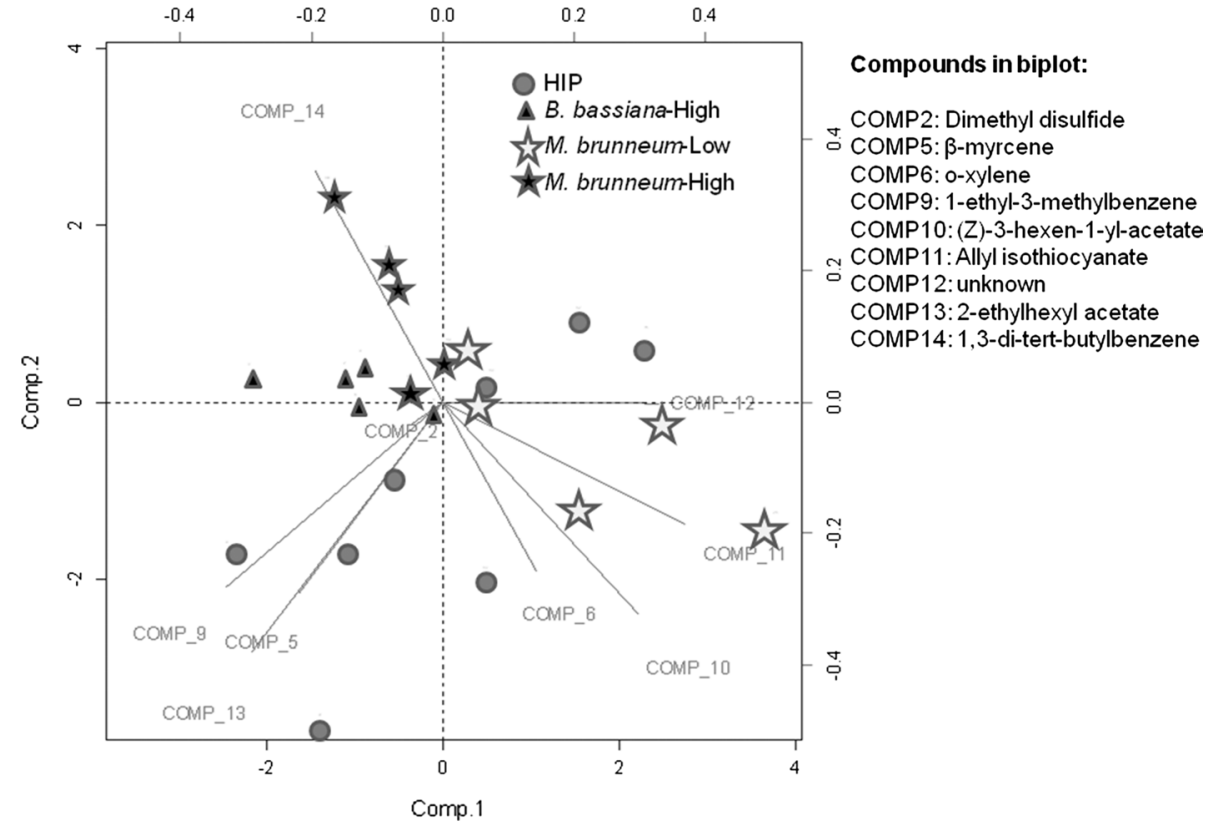

different volatile compounds (Table 3). From these compounds (COMP1-14), we only included COMP2, COMP5-6 and COMP9-14 in the PCA analysis, after discharging highly correlated compounds. Although PCA can deal with correlation (Zuur et al. 2007), those compounds which presumably measured the same underlying aspect of volatile emissions from the habitats were removed, assuming that including the redundant variables can cause the PCA to overemphasize their contribution.

The first three PCs accounted for $62.40 \%$ of the total variation of the data set. The biplot represents PC1 and PC2 (Fig. 3) and loadings of the first three PCA are presented in Online Resource 4. PC1 and PC2 were chosen because they clearly discriminated amongst the plant groups. As can be observed in Fig. 3, there are grouping clusters of samples. HIP and HIP with M. brunneum-Low can be differentiated from HIP M. brunneum-High and from B. bassiana-High. In the biplot the distance between the points shows the similarity in the observations (plants) based on the volatile compounds (lines). The projection of a point on a line represents the contribution of a certain compound to the volatile profile of that specific plant. Thus, the PCA loading table (Online Resource 4) indicates that 1,3-di-tertbutylbenzene was highly related to plants with $M$. brunneum-High while compounds such as dimethyl disulfide and 1-ethyl-3-methylbenzene are more representative of HIP. Finally, allyl isothiocyanate is located next to HIP with M. brunneum-Low.

The PerMANOVA showed that fungal species and fungal density affected the odor profile exhibited by HIP (PerMANOVA, $F_{3,22}=2.308, P<0.001$ ). Furthermore, Kruskal-Wallis tests followed by post hoc tests comparing the relative amounts of individual compounds emitted from HIP plants under the different treatments indicated significant differences for the compounds 1-ethyl-3-methylbenzene, unknown, and 1,3-di-tert-butylbenzene. However, only the latter compound differentiated high fungal densities of both fungal species from HIP and the plants with M. brunneum-Low (Table 3). This, combined with the separation in the PCA biplot, indicated differences between treatments.

\section{Discussion}

In this study we demonstrated that the belowground foraging parasitoid $T$. rapae was less attracted to host habitats infested by entomopathogenic fungi when searching for hosts. The fungi represented an IGP risk and we found that the lower attraction depended on fungal species and density. In addition, an earlier study showed that $T$. rapae females oviposit more in healthy hosts than in hosts infected by $M$. brunneum in a dual-choice experiment (Rännbäck et al. 2015). This indicates an ability of female $T$. rapae to evaluate host quality and IGP risks to their offspring. Furthermore, we found that when hosts were present in the habitat at high densities, this factor was more important for parasitoid attraction than presence of IG predators. Successful evaluation and response to cues indicating habitat quality, such as host density and presence of IGP risk, would allow T. rapae to select the most profitable host patch, leading to maximal reproductive success. Similarly, other studies on herbivore-natural enemy systems have reported that parasitoids make foraging decisions based on the risk of IGP 
(Raymond et al. 2000; Snyder and Ives 2008; Meisner et al. 2011; Velasco-Hernández et al. 2013), which may increase parasitoid fitness.

Spatial patchiness, described as heterogeneous environments in which prey and predators are spatially distributed (Hassell and May 1988), can influence the dynamics of host-parasitoid interactions, among other factors (Hassell 2000). Locating suitable hosts is critical for parasitoid fitness and responses to cues related to high host densities should be selected for. The distribution of $T$. rapae represents an example of parasitism with a direct densitydependent pattern. Under natural conditions, parasitoids with this type of functional response are aggregated in patches of high host density (Hassell and May 1973; Jones and Hassell 1988). Indeed, high host densities were most attractive to T. rapae females in the present study, which is in agreement with previous findings (Neveu et al. 2002; Hemachandra et al. 2007). Similarly, entomopathogenic fungi often display clumped distributions in the soil environment (Meyling and Eilenberg 2007). In such situations, the IGP risk in the particular patch may become important during host searches, as females could disperse from patches where IGP risks are present into alternative unexploited host patches. This decision to disperse by the IG prey, T. rapae, when encountering entomopathogenic fungi, and the absence of the IG predator in certain patches, could lead to a higher survival rate of the IG prey. This fundamental ecological principle (dispersal-competition tradeoff) has been applied to explain how spatial processes can promote coexistence between two competing species (Tilman 1994). Furthermore, besides increasing reproductive success, a preference for exploiting highly profitable host patches may also provide enemy-free space for $D$. radicum where populations are low. Both mechanisms will ultimately stabilize the host-parasitoid population dynamics (Vet 1999).

Our behavioral studies indicated that foraging T. rapae females reduce the IGP risk to themselves and their offspring through avoidance of host habitats with high densities of the IG predator M. brunneum. Avoidance responses to potential IGP risks to parasitoid offspring from entomopathogenic fungi have been observed in other studies with infected hosts (Brobyn et al. 1988; Fransen and van Lenteren 1993). However, in these studies no IGP risk was posed to the foraging parasitoid, as the fungal species tested were not pathogenic to adults. In contrast, our host-pathogen-parasitoid system included an IGP risk to both adult females and their offspring. Both fungal isolates used are pathogenic to adult $T$. rapae and to larvae of their host, $D$. radicum, with the $M$. brunneum isolate exhibiting the higher virulence (Rännbäck et al. 2015). Since the fungal species may infect the parasitoid larva inside the fungal infected host as well as the adult female parasitoid $T$. rapae during host foraging, both coincidental (IGP occurring when two species simultaneously attack a prey individual) and omnivorous (IGP occurring independently of prey consumption) IGP (Straub et al. 2008) respectively, are risks to T. rapae. If the foraging female fails to perceive an IGP risk and does not avoid fungal infested host habitats, coincidental IGP towards parasitoid larvae developing in infected hosts is likely. Furthermore, fungal infected T. rapae females can perceive an IGP risk to themselves and compensate for a reduced life expectancy through an increase in oviposition rate (Rännbäck et al. 2015).

Volatile collections from host habitats with IGP risk displayed different compound profiles, which correlated with the behavioral response observed for T. rapae in the bioassays.

The volatiles emitted from the plant-larvae host complex differed depending on whether the HIPs had been treated with fungus and on fungal density. The compound 1,3-di-tert-butylbenzene, an alkyl benzene, occurred only when high densities of either of the fungal isolates were present. Alkyl benzenes have previously been detected in fungal cultures (Jeleń and Wasowicz 1998). It has been reported that 1,3-di-tert-butylbenzene is released by $B$. bassiana (Crespo et al. 2008), and moreover, is emitted as the main volatile compound from mycelium cultures of another ascomycete, Tuber borchii Vitt (Tirillini et al. 2000). Its presence in our experimental habitats may indicate actively growing mycelium, but we cannot conclude whether 1,3-di-tert-butylbenzene originates from an interaction with cabbage roots, growth on larval cuticles, or a combination of these. Another interesting volatile is dimethyl disulfide, which we found in all treatments except $M$. brunneum-High. This compound is emitted by plants heavily infested with $D$. radicum larvae and it has been found to be attractive to the main predators of $D$. radicum in the field (Ferry et al. 2007). The absence of dimethyl disulfide in M. brunneum-High may have contributed to the reduced attraction of $T$. rapae to this treatment in our experiment. A substantial reduction in the feeding of $D$. radicum larvae could be evident due to the high virulence of the fungus, especially at high fungal densities (Rännbäck et al. 2015), and thus lead to lower emission rates of dimethyl disulfide. The females may use cues of host density and host quality, e.g., feeding status, fungal infection (IG predator), to detect differences between habitats and make foraging decisions. The composition and quantity of emitted volatiles constitute that cue.

Recently, entomopathogenic fungi were shown to be able to colonize the rhizosphere of several plants (Bruck 2010; Sasan and Bidochka 2012; Akutse et al. 2013; Razinger et al. 2014). The resulting mycelium could potentially be responsible for the production of volatile compounds such as 1,3-di-tert-butylbenzene, or induction 
of yet unknown compounds emitted by the plant. The ecological consequences of this plant-fungus interaction for other organisms are currently not fully understood. In contrast to our findings, Kepler and Bruck (2006) reported that a combination of pine tree roots and $M$. brunneum attracts larvae of the belowground herbivore Otiorhynchus sulcatus (F.) (Coleoptera: Curculionidae). The mechanisms behind this response are, however, unknown. Furthermore, there is increasing evidence that other root-colonizing fungi can induce changes in the profile of volatile organic compounds released by plants attacked by herbivores, resulting in the increased attraction of natural enemies (Schausberger et al. 2012; Battaglia et al. 2013; Pineda et al. 2013). However, in our system it remains to be identified which compounds are behaviorally active and whether their emission from the plant is caused by fungus, plant roots, host larvae or a combination of these.

Our findings show that host habitats infested with virulent fungi can influence the behavior of foraging parasitoids during host location. A dispersal strategy to visit patches unexploited by fungi may favor parasitoids by allowing them to forage in an enemy-free space and thus most likely achieve greater reproductive success. However, we also found that parasitoids encountering high-density host patches may ignore fungal presence in the habitat. These results emphasize the importance of understanding the behavior of foraging parasitoids when they visit patches with IG predators, since avoiding IGP risk is crucial for their survival and fitness.

Author contribution statement B. R., P. A., N. V. M. and L.-M. R. originally formulated the idea; B. R., P. A. and B. C. conceived and designed the experiments; L.-M. R. and N. V. M. performed insect and fungal methodologies; B. C. performed the experiments and developed the statistical models; M. B. and H. R. N. collaborated in chemical analysis; all authors wrote the manuscript.

\footnotetext{
Acknowledgments We thank the three anonymous reviewers for valuable comments on this paper, as well as Mira Rur for technical assistance, Dr. David Carrasco for statistical discussions, Dr. Gunda Thöming for her advice in the chemical analysis and Göran Nilsson for technical support in the Biotron facilities. We also thank Dr. Sebastien Dugravot, University of Rennes 1, for providing the initial strain of T. rapae and Dr. Rosemary Collier, University of Warwick, for providing the start culture of $D$. radicum. This study was supported by a PhD grant to L.-M. R. from the Swedish Research Council for Environment, Agricultural Sciences and Spatial Planning (FORMAS; project number 2009-5824-14,994-47) and the Swedish University of Agricultural Sciences (SLU). Analysis performed at the Norwegian Institute for Agricultural and Environmental Research (Bioforsk) was financially supported by the Norwegian Research Council (project number 186903). The English version of this manuscript was revised by Mary McAfee.
}

Open Access This article is distributed under the terms of the Creative Commons Attribution 4.0 International License (http://creativecommons.org/licenses/by/4.0/), which permits unrestricted use, distribution, and reproduction in any medium, provided you give appropriate credit to the original author(s) and the source, provide a link to the Creative Commons license, and indicate if changes were made.

\section{References}

Akutse KS, Maniania NK, Fiaboe KKM, Van den Berg J, Ekesi S (2013) Endophytic colonization of Vicia faba and Phaseolus vulgaris (Fabaceae) by fungal pathogens and their effects on the life-history parameters of Liriomyza huidobrensis (Diptera: Agromyzidae). Fungal Ecol 6:293-301

Anderson MJ (2001) A new method for non-parametric multivariate analysis of variance. Austral Ecol 26:32-46

Battaglia D (2013) Tomato below ground-above ground interactions: Trichoderma longibrachiatum affects the performance of Macrosiphum euphorbiae and its natural antagonists. Mol Plant Microbe Int 26:1249-1256. doi:10.1094/mpmi-02-13-0059-r

Baverstock J, Alderson PG, Pell JK (2005) Influence of the aphid pathogen Pandora neoaphidis on the foraging behaviour of the aphid parasitoid Aphidius ervi. Ecol Entomol 30:665-672. doi:10.1111/j.0307-6946.2005.00744.x

Baverstock, Roy HE, Pell JK (2010) Entomopathogenic fungi and insect behaviour: from unsuspecting hosts to targeted vectors. Biocontrol 55:89-102. doi:10.1007/s10526-009-9238-5

Brobyn PJ, Clark SJ, Wilding N (1988) The effect of fungus infection of Metopolophium dirhodum (Hom.: Aphididae) on the oviposition behaviour of the aphid parasitoid Aphidius rhopalosiphi (Hym.: Aphidiidae). Entomophaga 33:333-338

Brooks WM (1993) Host-parasitoid-pathogen interactions. In: Beckage NE, Thompson SN, Federici BA (eds) Parasites and pathogens of insects. Academic Press, San Diego, pp 231-272

Brown PE, Anderson M (1999) Factors affecting ovipositor probing in Trybliographa rapae, a parasitoid of the cabbage root fly. Entomol Exp Appl 93:217-225. doi:10.1046/j.1570-7458.1999.00581.x

Bruck D (2010) Fungal entomopathogens in the rhizosphere. Biocontrol 55:103-112. doi:10.1007/s10526-009-9236-7

Chandler D, Davidson G (2005) Evaluation of entomopathogenic fungus Metarhizium anisopliae against soil-dwelling stages of cabbage maggot (Diptera: Anthomyiidae) in glasshouse and field experiments and effect of fungicides on fungal activity. J Econ Entomol 98:1856-1862. doi:10.1603/0022-0493-98.6.1856

Connor EF, Cargain MJ (1994) Density-related foraging behaviour in Closterocerus tricinctus, a parasitoid of the leaf-mining moth, Cameraria hamadryadella. Ecol Entomol 19:327-334. doi:10.1111/j.1365-2311.1994.tb00249.x

Crespo R, Pedrini N, Juarez MP, Dal Bello GM (2008) Volatile organic compounds released by the entomopathogenic fungus Beauveria bassiana. Microbiol Res 163:148-151. doi:10.1016/j. micres.2006.03.013

Dicke M, Baldwin IT (2010) The evolutionary context for herbivoreinduced plant volatiles: beyond the cry for help. Trends Plant Sci 15:167-175. doi:10.1016/j.tplants.2009.12.002

Dicke M, Grostal P (2001) Chemical detection of natural enemies by arthropods: an ecological perspective. Annu Rev Ecol Syst 32:123. doi:10.1146/annurev.ecolsys.32.081501.113951

Ferry A (2007) Identification of a widespread monomolecular odor differentially attractive to several Delia radicum grounddwelling predators in the field. J Chem Ecol 33:2064-2077. doi:10.1007/s10886-007-9373-3 
Finch S (1989) Ecological considerations in the management of a Delia pest species in vegetable crops. Annu Rev Entomol 34:117-137

Finch S, Coaker TH (1969) A method for the continuous rearing of the cabbage root fly Erioischia brassicae (Bch.) and some observations on its biology. Bull Entomol Res 58:619-627. doi: $10.1017 / \mathrm{S} 0007485300057345$

Fransen JJ, van Lenteren JC (1993) Host selection and survival of the parasitoid Encarsia formosa on greenhouse whitefly, Trialeurodes vaporariorum in the presence of hosts infected with the fungus Aschersonia aleyrodis. Entomol Exp Appl 69:239-249

Furlong MJ, Pell JK (2005) Interactions between entomopathogenic fungi and arthropod natural enemies. In: Vega FE, Blackwell M (eds) Insect-fungal associations: ecology and evolution. Oxford University Press, New York, pp 51-73

Girling RD, Stewart-Jones A, Dherbecourt J, Staley JT, Wright DJ, Poppy GM (2011) Parasitoids select plants more heavily infested with their caterpillar hosts: a new approach to aid interpretation of plant headspace volatiles. Proc R Soc B Biol Sci 278:26462653. doi: $10.1098 / \mathrm{rspb} .2010 .2725$

Guerrieri E, Lingua G, Digilio MC, Massa N, Berta G (2004) Do interactions between plant roots and the rhizosphere affect parasitoid behaviour? Ecol Entomol 29:753-756. doi:10.1111/j.0307-6946.2004.00644.x

Hassell M (2000) Host-parasitoid population dynamics. J Anim Ecol 69:543-566

Hassell MP, May RM (1973) Stability in insect host-parasite models. J Anim Ecol 42:693-726

Hassell MP, May RM (1988) Spatial heterogeneity and the dynamics of parasitoid-host systems. Ann Zool Fenn 25:55-61

Hemachandra KS, Kuhlmann U, Mason PG, Holliday NJ (2007) Spatial patterns of Trybliographa rapae parasitism of Delia radicum larvae in oilseed rape and cauliflower. J Appl Entomol 131:338346. doi:10.1111/j.1439-0418.2007.01184.x

Jeleń H, Wasowicz E (1998) Volatile fungal metabolites and their relation to the spoilage of agricultural commodities. Food Rev Int 14:391-426. doi:10.1080/87559129809541170

Jones TH, Hassell MP (1988) Patterns of parasitism by Trybliographa rapae, a cynipid parasitoid of the cabbage root fly, under laboratory and field conditions. Ecol Entomol 13:309-317. doi:10.1111/j.1365-2311.1988.tb00361.x

Jönsson M, Lindkvist A, Anderson P (2005) Behavioural responses in three ichneumonid pollen beetle parasitoids to volatiles emitted from different phonological stages of oilseed rape. Entomol Exp Appl 115:363-369

Kepler RM, Bruck DJ (2006) Examination of the interaction between the black vine weevil (Coleoptera: Curculionidae) and an entomopathogenic fungus reveals a new tritrophic interaction. Environ Entomol 35:1021-1029. doi:10.1603/0046-225X-35.4.1021

Lord JC (2001) Response of the wasp Cephalonomia tarsalis (Hymenoptera: Bethylidae) to Beauveria bassiana (Hyphomycetes: Moniliales) as free conidia or infection in its host, the sawtoothed grain beetle, Oryzaephilus surinamensis (Coleoptera: Silvanidae). Biol Control 21:300-304

Mburu DM, Maniania NK, Hassanali A (2013) Comparison of volatile blends and nucleotide sequences of two Beauveria bassiana isolates of different virulence and repellency towards the termite Macrotermes Michealseni. J Chem Ecol 39:101-108. doi:10.1007/s10886-012-0207-6

Meisner M, Harmon JP, Harvey CT, Ives AR (2011) Intraguild predation on the parasitoid Aphidius ervi by the generalist predator Harmonia axyridis: the threat and its avoidance. Entomol Exp Appl 138:193-201

Meyling NV, Eilenberg J (2007) Ecology of the entomopathogenic fungi Beauveria bassiana and Metarhizium anisopliae in temperate agroecosystems: potential for conservation biological control. Biol Control 43:145-155

Meyling NV, Pell JK (2006) Detection and avoidance of an entomopathogenic fungus by a generalist insect predator. Ecol Entomol 31:162-171. doi:10.1111/j.0307-6946.2006.00781.x

Montgomery DC, Peck EA (1992) Introduction to linear regression analysis. Wiley, New York

Nakashima Y, Birkett MA, Pye BJ, Pickett JA, Powell W (2004) The role of semiochemicals in the avoidance of the seven-spot ladybird, Coccinella septempunctata, by the aphid parasitoid, Aphidius ervi. J Chem Ecol 30:1103-1116

Neveu N, Kacem N, Nenon JP (1996) A method for rearing Trybliographa rapae W. on Delia radicum L. Bull OILB/SROP 19:173-178

Neveu N, Grandgirard J, Nenon JP, Cortesero AM (2002) Systemic release of herbivore-induced plant volatiles by turnips infested by concealed root-feeding larvae Delia radicum L. J Chem Ecol 28:1717-1732. doi:10.1023/A:1020500915728

Nilsson U, Eriksson A, Rämert B, Anderson P (2012) Male and female Trybliographa rapae (Hymenoptera: Figitidae) behavioral responses to food plant, infested host plant and combined volatiles. Arthropod-Plant Interact 6:251-258

Oksanen J (2013) Vegan: Community Ecology package R package version $2.0-10$

Ormond EL, Thomas APM, Pell JK, Freeman SN, Roy HE (2011) Avoidance of a generalist entomopathogenic fungus by the ladybird, Coccinella septempunctata. FEMS Microbiol Ecol 77:229237. doi:10.1111/j.1574-6941.2011.01100.x

Pierre J-S, Green RF (2008) A Bayesian approach to optimal foraging in parasitoids. In: Behavioral ecology of insect parasitoids. Blackwell, Oxford, pp 357-383

Pineda ANA, Soler R, Weldegergis BT, Shimwela MM, Van Loon JJA, Dicke M (2013) Non-pathogenic rhizobacteria interfere with the attraction of parasitoids to aphid-induced plant volatiles via jasmonic acid signalling. Plant Cell Environ 36:393-404. doi:10.1111/j.1365-3040.2012.02581.x

Plummer M (2008) Penalized loss functions for Bayesian model comparison. Biostatistics 9:523-539. doi:10.1093/biostatistics/kxm049

Polis GA, Myers CA, Holt RD (1989) The ecology and evolution of intraguild predation: potential competitors that eat each other. Annu Rev Ecol Syst 20:297-330. doi:10.1146/annurev. es.20.110189.001501

R Core Team (2012) R: a language and environment for statistical computing. R Foundation for Statistical Computing, Vienna

Rännbäck L-M, Cotes B, Anderson P, Rämert B, Meyling NV (2015) Mortality risk from entomopathogenic fungi affects oviposition behavior in the parasitoid wasp Trybliographa rapae. J Invertebr Pathol 124:78-86. doi:10.1016/j.jip.2014.11.003

Raymond B, Darby AC, Douglas AE (2000) Intraguild predators and the spatial distribution of a parasitoid. Oecologia 124:367-372. doi: $10.1007 / \mathrm{s} 004420000396$

Razinger J (2014) Direct plantlet inoculation with soil or insect-associated fungi may control cabbage root fly maggots. J Invertebr Pathol 120:59-66. doi:10.1016/j.jip.2014.05.006

Roitberg BD, Zimmermann K, Hoffmeister TS (2010) Dynamic response to danger in a parasitoid wasp. Behav Ecol Sociobiol 64:627-637

Rosenheim JA, Kaya HK, Ehler LE, Marois JJ, Jaffee BA (1995) Intraguild predation among biological control agents: theory and evidence. Biol Control 5:303-335

Roy HE, Pell JK (2000) Interactions between entomopathogenic fungi and other natural enemies: implications for biological control. Biocontrol Sci Technol 10:737-752

Roy HE, Steinkraus DC, Eilenberg J, Hajek AE, Pell JK (2006) Bizarre interactions and endgames: entomopathogenic fungi and their arthropod hosts. Annu Rev Entomol 51:331-357 
Sasan RK, Bidochka MJ (2012) The insect-pathogenic fungus Metarhizium robertsii (Clavicipitaceae) is also an endophyte that stimulates plant root development. Am J Bot 99:101-107. doi:10.3732/ajb.1100136

Schausberger P, Peneder S, Jürschik S, Hoffmann D (2012) Mycorrhiza changes plant volatiles to attract spider mite enemies. Funct Ecol 26:441-449. doi:10.1111/j.1365-2435.2011.01947.x

Snyder WE, Ives AR (2008) Behavior influences whether intra-guild predation disrupts herbivore suppression by parasitoids. In: Wajenberg E, Bernstein C, Van Alphen J (eds) Behavioral ecology of insect parasitoids. Blackwell, Malden, pp 71-91

Soler R, Bezemer TM, Harvey JA (2013) Chemical ecology of insect parasitoids in a multitrophic above- and below-ground context. In: Wajnberg E, Colazza S (eds) Chemical ecology of insect parasitoids. Wiley-Blackwell, Chichester, pp 64-85

Spiegelhalter DJ, Best NG, Carlin BP, Van Der Linde A (2002) Bayesian measures of model complexity and fit. J R Stat Soc B 64:583-639

Straub CS, Finke DL, Snyder WE (2008) Are the conservation of natural enemy biodiversity and biological control compatible goals? Biol Control 45:225-237

Su Y-S, Yajima M (2012) R2jags: a package for running jags from R. $\mathrm{R}$ package version $0.03-08$

Thöming G, Norli H, Saucke H, Knudsen G (2014) Pea plant volatiles guide host location behaviour in the pea moth. Arthropod Plant Interact 8:109-122. doi:10.1007/s11829-014-9292-5

Tilman D (1994) Competition and biodiversity in spatially structured habitats. Ecology 75:2-16. doi:10.2307/1939377

Tirillini B, Verdelli G, Paolocci F, Ciccioli P, Frattoni M (2000) The volatile organic compounds from the mycelium of Tuber borchii Vitt. Phytochemistry 55:983-985
Velasco-Hernández MC, Ramirez-Romero R, Cicero L, Michel-Rios C, Desneux N (2013) Intraguild predation on the whitefly parasitoid Eretmocerus eremicus by the generalist predator Geocoris punctipes: a behavioral approach. PLoS One 8:e80679

Vet LM (1999) From chemical to population ecology: infochemical use in an evolutionary context. J Chem Ecol 25:31-49. doi:10.10 23/A:1020833015559

Vet LEM, Dicke M (1992) Infochemical use by natural enemies of herbivores in a tritrophic context. Annu Rev Entomol $37: 141-172$

Waage JK (1983) Aggregation in field parasitoid populations: foraging time allocation in a population of Diadegma (Hymenoptera, Ichneumonidae). Ecol Entomol 8:447-454

Wäschke N, Meiners T, Rostas M (2013) Foraging strategies of parasitoids in complex chemical environments. In: Wajnberg E, Colazza S (eds) Chemical ecology of insect parasitoids. Wiley, Chichester, pp 38-63

Weisser WW, Houston A, Völkl W (1994) Foraging strategies in solitary parasitoids: the trade-off between female and offspring mortality risks. Evol Ecol 8:587-597. doi:10.1007/BF01237843

Wishart F, Monteith E (1954) Trybliographa rapae (Westw.) (Hymenoptera: Cynipidae). A parasite of Hylemya spp. (Diptera: Anthomyiidae). Can Entomol 86:145-154

Zuur AF, Ieno EN, Smith GM (2007) Analysing ecological data. Springer, New York

Zuur AF, Ieno EN, Elphick CS (2010) A protocol for data exploration to avoid common statistical problems. Methods Ecol Evol 1:3-14. doi:10.1111/j.2041-210X.2009.00001.x 